\title{
The Upper Nasal Space-A Novel Delivery Route Ideal for Central Nervous System Drugs
}

John Hoekman, Sutapa Ray, Sheena K Aurora, and Stephen B Shrewsbury

Impel NeuroPharma, Inc., Seattle, WA, USA

$\mathrm{S}$ olid oral dosage forms account for up to $75 \%$ of prescriptions, but their effectiveness may be limited in some patients, and inappropriate for others. While injectable formulations may be an option for some central nervous system (CNS) disorders, injections are poorly accepted by most patients, except in an urgent or emergency situation. Thus, a need exists for non-oral dosage formulations as alternatives to treat conditions such as acute migraine, acute agitation or irritability, and OFF episodes in Parkinson's disease, where oral forms are not optimal. Nasal drug delivery has been underutilized, provides a more rapid onset of activity, avoids gastrointestinal (GI) tract degradation and first-pass hepatic metabolism, achieves faster therapeutic plasma levels, may reduce GI adverse events, and provides patient convenience and ease-of-use. However, drug loss from the nose, which may lead to variability in absorption, has been one of several issues limiting use of nasal delivery. An innovative, non-oral drug/device delivery system that targets the upper nasal space is in late-stage clinical development for treating CNS disorders and its availability is expected to expand over the next few years, providing welcome additions for the treatment of CNS disorders in patients where oral dosage forms are not optimal.

\section{Keywords}

Acute migraine, agitation, nasal, non-oral drug delivery, Parkinson's disease

Disclosures: John Hoekman, Sutapa Ray, Sheena K Aurora, and Stephen B Shrewsbury are full-time employees of and own stock in, Impel NeuroPharma, Inc.

Acknowledgments: The authors would like to acknowledge the editorial assistance of Richard S Perry, PharmD, in the development of this manuscript, which was supported by Impel NeuroPharma Inc., Seattle, WA, USA.

\section{Review Process: Double-blind peer review.}

Compliance with Ethics: This article involves a review of the literature and did not involve any studies with human or animal subjects performed by any of the authors.

Authorship: The named authors meet the International Committee of Medical Journal Editors (ICMJE) criteria for authorship of this manuscript, take responsibility for the integrity of the work as a whole, and have given final approval for the version to be published. Access: This article is freely accessible at touchNEUROLOGY.com (c) Touch Medical Media 2020.

Received: March 16, 2020

Accepted: May 6, 2020

Published Online: July 10, 2020

Citation: US Neurology. 2020:16(1):25-31

Corresponding Author: Stephen B Shrewsbury, Impel NeuroPharma, Inc., 201 Elliott Avenue West, Suite 260, Seattle, WA 98119, USA.

E: sshrewsbury@impelnp.com

Support: No funding was received for

the publication of this article.
Treatment of acute central nervous system (CNS) conditions requires effective drugs that can provide rapid onset of effect, consistent blood levels, ease-of-use for patient or caregiver, and acceptable tolerability. Solid oral dosage forms account for up to $75 \%$ of prescriptions from healthcare providers. ${ }^{1}$ They are less expensive and easier to manufacture than drug-device combination products, offer good stability, may be developed with controlled-release properties, are non-invasive, may provide accurate dosing, and are both convenient and easy to use for the patient., However, oral delivery may result in slow absorption and onset of effect, ${ }^{3}$ side effects due to high systemic drug levels, ${ }^{4}$ and possible interactions with food or other orally administered drugs. ${ }^{5}$ Some CNS disorders, such as migraine, are associated with high rates of nausea and vomiting, that can further impair oral absorption, ${ }^{1,2,6,7}$ and an often under-appreciated gastrointestinal (GI) dysmotility both within and between migraine episodes. ${ }^{8,9}$ Further, some oral triptans have lower oral bioavailability $\leq 50 \%$ (eletriptan, frovatriptan, rizatriptan, sumatriptan, zolmitriptan) ${ }^{10}$ and slow absorption, resulting in delayed peak plasma concentration $\left(T_{\max }\right)$ ranging from 1.2-2.0 hours, and slow onset of action ${ }^{10}$ or a lack of effect if the "triptan window" is missed."

Among patients with Parkinson's disease, gastric stasis often occurs and food intake may interfere with oral medication absorption. ${ }^{12}$ Indeed, oral dosing may be limited by the concomitant presence of gastroparesis, swallowing difficulties or dysphagia, that occur in up to two-thirds of patients. ${ }^{1,2}$ Up to one-third of the population experiences swallowing difficulties during their lifetime, ${ }^{2}$ and even among the general population, 1-16\% experience dysphagia, rising to $>60 \%$ of those in nursing homes who experience difficulty swallowing solid oral dosage forms. ${ }^{6}$

While injectable formulations may be an option for some CNS disorders, injections are poorly accepted by most patients, especially those with underlying mental illness; ${ }^{\circ 3}$ may weaken patient-physician trust; and may require healthcare professional administration. Further, the need for restraint during administration may lead to nursing staff and patient injuries. ${ }^{14}$ Thus, a need exists for non-injected, non-oral dosage formulations with the efficiency of an injectable, as alternatives for treating many acute CNS conditions.

\section{Nasal drug delivery systems for central nervous system disorders}

Nasal drug delivery has the potential to provide a more rapid onset of activity; avoids degradation in the GI tract and first-pass hepatic metabolism; is associated with a low risk for Gl adverse events; can be administered independent of meals, potentially by a caregiver; and provides patient convenience and ease-of-use. ${ }^{15}$ Several nasal delivery systems are commercially available, with several more, 
including one targeting the previously unutilized upper nasal space, in clinical development.

In addition, a growing body of research is investigating the possibility of direct access of small-, and especially of large-molecule drugs via olfactory and trigeminal pathways to the CNS using nasal delivery. ${ }^{16,17}$ This may allow the circumvention of the blood-brain barrier and blood-cerebrospinal fluid barrier. ${ }^{17}$ The nose-to-brain pathway in animals was first confirmed in 1985 using wheat germ agglutinin-horseradish peroxidase, ${ }^{18}$ but had been known since the 1930s as the route for poliomyelitis virus ingress, ${ }^{19}$ later for vesicular stomatitis virus, ${ }^{20}$ and later still for metals.. ${ }^{21}$ More recently, several early development programs have shown the ability of nasally delivered neural stem cells to migrate through the cribriform plate at the ceiling of the upper nasal space and thence into the olfactory bulb or into the cerebrospinal fluid. ${ }^{22,23}$ While this research is encouraging, we will limit this review to the emerging clinical data from three different clinical programs delivering established small-molecule CNS drugs to the previously unutilized upper nasal space. Early and consistent systemic blood levels have been reported making this device a potentially useful future option for consideration. Now that systemic blood levels due to delivery to the upper nasal space has been demonstrated, predictably and repeatedly, in humans, the way is open for future non-invasive programs to utilize this route with small- or large-molecule new chemical entities, oligonucleotides, or even cells.

\section{Traditional nasal delivery targeting the lower nasal cavity}

The potential advantages of nasal drug delivery to treat acute migraine have been recognized for many years, including avoidance of $\mathrm{Gl}$ absorption, decreased side effects, and more rapid onset compared with oral medications. ${ }^{24}$ Several drugs using this route have been approved, with more in development, including dihydroergotamine (DHE) mesylate (Migranal ${ }^{\circledR}$ [Bausch Health, Laval, Canada] approved in 1996 and new liquid [INP104] and powder [STS101] products in late clinical development), sumatriptan (Imitrex ${ }^{\circledR}$ [GlaxoSmithKline, Brentford, UK] in 1992, Onzetra Xsail ${ }^{\circledR}$ [Currax Pharmaceuticals, LLC, Morristown, NJ, USA] in 2016, and

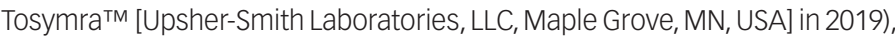
and zolmitriptan (Zomig [Amneal Pharmaceuticals, Bridgewater Township, NJ, USA] in 2003). Yet according to the 2009 American Migraine Prevalence and Prevention study, at least $40 \%$ of patients have at least one unmet need from their medication, and only $\sim 5.5$ million (of the estimated pool of 39 million) patients in the USA receive a regular preventive medicine. ${ }^{25}$ Migraine remains a major cause of disability and lost productivity, especially for otherwise healthy adults during their most productive years. ${ }^{26}$

In addition, potential limitations of traditional nasal drug administration include rapid elimination from the nasal space due to dripping from the front of the nose, or down the back of the throat, ${ }^{16}$ exacerbated by improper administration technique; mucociliary clearance; and lower nasal space architecture. The respiratory mucosa of the lower nasal space is prone to edema and inflammation, or variability in mucous layer cover as a result of infections or allergy, leading to variable drug absorption..$^{16}$ In addition, the respiratory mucosa may be subject to local discomfort and irritation, ${ }^{15}$ and drug lost to the nasopharynx can result in taste disturbance. ${ }^{27}$

\section{Upper nasal drug delivery}

While the nasal space provides an option for delivering drugs to the systemic circulation, most (traditional) nasally administered drugs reach
Figure 1: Target delivery area and powder precision olfactory delivery system (INP105, INP103, and INP107)

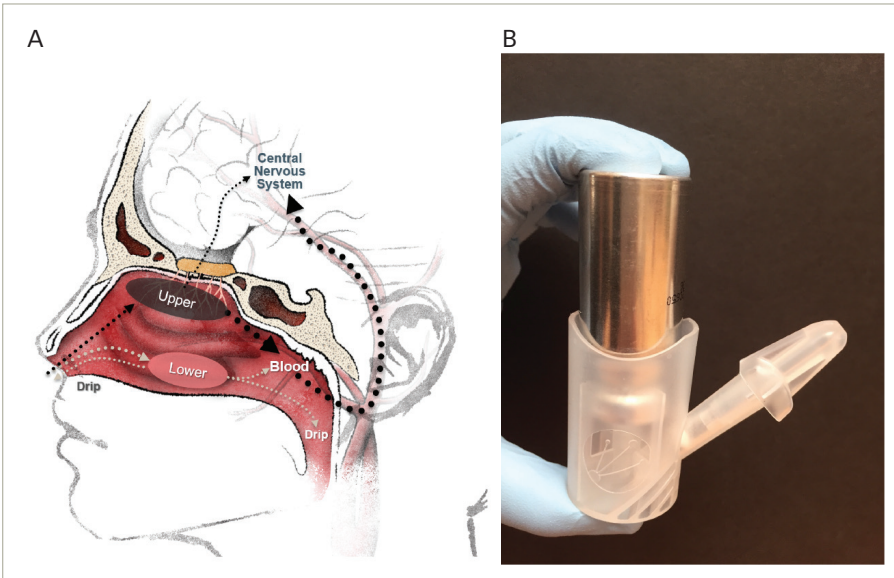

A. Diagram of a sagital section through the nose showing the typical target area for nasal sprays (lower) and POD (upper) systems; B. The loaded POD research device las used in INP105-101 and INP103-201).

INP105 = olanzapine delivered by POD; INP103 = levodopa delivered by POD;

INP107 = carbidopa/levodopa delivered by POD; POD = precision olfactory delivery.

only the vestibule with its squamous epithelium or lower nasal space and turbinates, where absorption via the respiratory epithelium is variable and plasma drug levels are inconsistent..$^{28}$ Due to its complex architecture, drugs delivered here, by standard nasal devices such as droppers, sprays or pumps, often deposit $<5 \%$ of active drug into the upper nasal space where absorption is greater, but can sometimes be improved by active, forced sniff.29 Delivery to the olfactory epithelium-lined, highly vascular upper nasal space (Figure 1A) provides faster systemic absorption, resulting in therapeutic drug levels and systemic drug effects. ${ }^{28}$

\section{Precision olfactory delivery}

Impel NeuroPharma developed the Precision Olfactory Delivery, or POD ${ }^{\circledR}$, nasal drug delivery platform, which utilizes the rich vasculature found in the olfactory region of the upper nasal space to provide consistent and predictable drug delivery and improve bioavailability (Figure 1A). ${ }^{28,30}$ The thickness of the olfactory mucosa in the upper nasal space remains relatively constant to allow the olfactory neurites that penetrate through this mucosa to sense the environment. The drug is less prone to be lost from this space to pharynx or nares; thus consistent drug delivery to this previously overlooked area should provide consistent drug levels and hence clinical response. ${ }^{28,30}$

The POD system is a handheld, manually-actuated, gas-propelled, administration device designed to deliver active drug specifically to the upper nasal space (Figure 1B). The proprietary nozzle design allows for a narrow-targeted plume to pass beyond the nasal valve to reach the expansive surface area of the upper and middle turbinates and olfactory epithelium. The biphasic emission of the propellant launches the drug, and then pushes it to the farthest reaches of the nasal space. By maintaining the hydrofluoroalkane propellant and the liquid, or powder drug, separate until the time of delivery, the POD overcomes the manufacturing challenges of maintaining dose uniformity and drug stability when active drug is suspended in the hydrofluoroalkane propellant. The system can overcome the limitations of older nasal delivery systems by utilizing metered, propellant-powered delivery without the need to coordinate 
Figure 2: Mean plasma DHE concentration following single doses of INP104 ${ }^{27}$

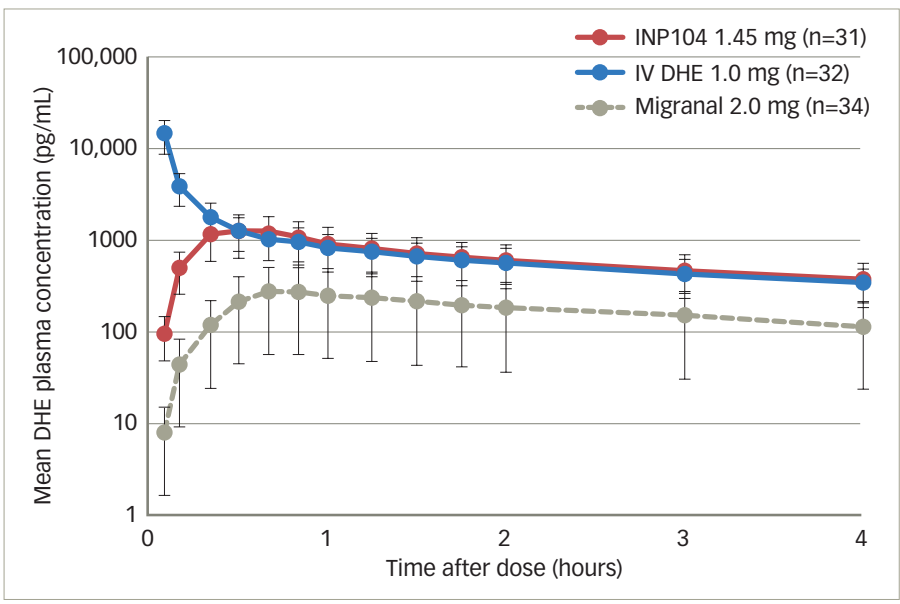

$D H E=$ dihydroergotamine mesylate; $I N P 104=$ DHE delivered by POD; IV = intravenous; $P O D=$ precision olfactory delivery..

breathing or keep the head in a specific orientation, and can be easily administered by the patients themselves, or even by a caregiver. Currently, POD technology is being investigated to deliver $\mathrm{DHE}_{1}{ }^{27,31}$ olanzapine (OLZ), ${ }^{32}$ and a combination of carbidopa plus levodopa ${ }^{33}$ to treat acute migraine, acute agitation, and OFF episodes in patients with Parkinson's disease, respectively.

\section{Clinical data}

Acute treatment of migraine

INP104-dihydroergotamine mesylate (in development)

Delivery of DHE using POD technology (INP104) was evaluated in an open-label, randomized, three-period, three-way crossover study, in 38 healthy subjects (ClinicalTrials.gov identifier: NCT03401346). ${ }^{27}$ Subjects received single doses of INP104 $1.45 \mathrm{mg}$ (treatment A), intravenous (IV) DHE $1.0 \mathrm{mg}$ (treatment B), and DHE nasal spray $2.0 \mathrm{mg}$ (treatment $\mathrm{C}$ ) in one of six sequences (treatments: $A B C, A C B, B A C, B C A, C A B, C B A$ ). Whilst INP104 avoided the undesirable high-peak plasma DHE concentration seen with IV administration, it generated similar plasma levels by 20 minutes and comparable exposure (area under the curve [AUC]) (Figure 2). ${ }^{27}$ INP104 achieved a four-fold increase in DHE $C_{\max }$ and a three-fold increase in DHE AUC compared with liquid DHE nasal spray (Migranal), using the identical formulation but $<75 \%$ of the dose. Variability in $\mathrm{C}_{\max }$ and AUC was substantially reduced with INP104. The incidence of any treatment-emergent adverse event was 19.4\% with INP104, 34.4\% with IV DHE, and $11.8 \%$ with DHE nasal spray. Treatment-related nausea was only reported with IV DHE (three subjects) and DHE nasal spray (one subject), despite metoclopramide premedication. Mild nasal discomfort occurred in one subject each with INP104 and liquid DHE nasal spray. Drug leakage from the nose or into the nasopharynx was reported by $32.3 \%$ with INP104, and 76.5\% with nasal spray. INP104 provides a novel nasal delivery system with a pharmacokinetic (PK) profile that is comparable to IV DHE from 20 minutes, with greater consistency than the nasal spray and in an easy-to-use system.

Results from a 360-patient phase III study with INP104 (STOP-301; ClinicalTrials.gov identifier: NCT03557333), assessing the safety and tolerability of chronic, intermittent use over 24 weeks, with a subset over
52 weeks, are expected in 2020. ${ }^{31}$ Nasal mucosal integrity and olfactory function are being evaluated with upper nasal endoscopy and the University of Pennsylvania Smell Identification Test (UPSIT), respectively. STOP-301, enrolling patients with a mean baseline migraine frequency of 4.7 episodes/month, is an open-label safety study utilizing a daily e-diary to assess exploratory efficacy, healthcare utilization and quality of life, and will represent the largest dataset of repeat, long-term DHE use in acute migraine when published. The data was collected both during the 28-day screening period, as well as for every day and with every migraine during the 24- or 52-week treatment periods.

Interim data on the acceptability of the product, ${ }^{34}$ reported results for a series of questions asked of 164 subjects who had then completed the 24-week study. Patients reported agreement or strong agreement that INP104 was easy to use in $90 \%$ of cases. Other questions asked were: did INP104 allow you to return to normal faster? Did INP104 consistently treat your migraines? Did INP104 work faster than your previous treatment? Did INP104 stop your migraines from coming back? Was INP104 easy to use? Answers to these questions were all scored: strongly agree; agree; neutral; disagree; or strongly disagree, and all provided encouraging data.

\section{Acute agitation \\ INP105-olanzapine (in development)}

Acute agitation is common in patients with schizophrenia and bipolar disorder, ${ }^{35}$ as well as other mental health disorders such as post-traumatic stress disorder, Alzheimer's dementia, and neurodevelopmental conditions, for example, Down's syndrome or autism spectrum disorder, where it may be referred to as "irritability". ${ }^{36}$ OLZ intramuscular (IM) is often used to treat acute agitation because of a shorter $\mathrm{T}_{\max }$ leading to more rapid onset of efficacy than oral administration, and a lower risk of extrapyramidal adverse effects than first-generation antipsychotics, but requires patient cooperation or restraint, is invasive, and can be painful. ${ }^{14}$ Injection may risk injury, loss of doctor-patient trust, and result in psychiatric boarding; whereas oral products have slower onset of effect, often requiring observation of the medicated patient for a sustained period of time, until their agitation resolves, ${ }^{36}$ which may be impractical. While non-pharmacological management is preferred, access to the appropriate staff to conduct it, time and location to deliver it, and other operational issues often make it hard to deliver in practice, and effective, rapid-acting, non-injected options are lacking.

In 2018, the Centers for Disease Control and Prevention determined that autism spectrum disorder may affect 1 in 37 boys and 1 in 151 girls. ${ }^{37}$ There are currently two oral antipsychotics approved for autism-associated agitation and irritability, yet interestingly children with autism have an eight-fold higher chance of chronic GI disorders, ${ }^{38}$ and chronic dosing with these drugs to reduce the incidence of agitation is hampered by tolerability issues. A rapidly-effective therapy for relief of agitation or irritability in these patients is much needed, especially non-oral.

\section{Precision olfactory delivery olanzapine (in development)}

A nasally-administered, fast-acting, well-tolerated, OLZ formulation would provide an alternative to IM or oral forms. INP105 is a drug-device combination product consisting of a powder formulation of OLZ delivered by $\mathrm{POD}^{32}$ at a dose of $5 \mathrm{mg}$ with each actuation, taking approximately one-tenth of a second. INP105 offers non-invasive delivery of OLZ into the upper nasal space, enabling rapid systemic absorption without an injection. 
Table 1: Treatment-emergent adverse events reported in the phase I (two-period, double-blind, placebo-controlled) SNAP 101 trial conducted in healthy volunteers ${ }^{32}$

\begin{tabular}{|c|c|c|c|c|c|c|c|}
\hline & \multicolumn{7}{|c|}{ Number (\%) of subjects } \\
\hline & \multicolumn{3}{|c|}{ Period 1 dosing } & \multicolumn{4}{|c|}{ Period 2 dosing } \\
\hline & $\begin{array}{l}\text { OLZ IM } \\
5 \mathrm{mg} \\
(\mathrm{n}=20) \\
\end{array}$ & $\begin{array}{l}\text { OLZ IM } \\
10 \mathrm{mg} \\
(\mathrm{n}=2)\end{array}$ & $\begin{array}{l}\text { OLZ ODT } \\
10 \mathrm{mg} \\
(\mathrm{n}=18)\end{array}$ & $\begin{array}{l}\text { INP105 } \\
5 \mathrm{mg} \\
(\mathrm{n}=10)\end{array}$ & $\begin{array}{l}\text { INP105 } \\
10 \mathrm{mg} \\
(\mathrm{n}=9)\end{array}$ & $\begin{array}{l}\text { INP105 } \\
15 \mathrm{mg} \\
(\mathrm{n}=8)\end{array}$ & $\begin{array}{l}\text { Placebo } \\
(\mathrm{n}=10)\end{array}$ \\
\hline Any adverse event & $18(90.0)$ & $2(100.0)$ & 15 (83.3) & $8(80.0)$ & $6(66.7)$ & $6(75.0)$ & $1(10.0)$ \\
\hline Dizziness & $4(20.0)$ & $1(50.0)$ & 0 & $1(10.0)$ & $2(22.2)$ & 0 & 0 \\
\hline Fatigue & 0 & 0 & 0 & $2(20.0)$ & 0 & 0 & 0 \\
\hline Headache & $2(10.0)$ & 0 & $6(33.3)$ & $1(10.0)$ & 0 & $1(12.5)$ & 0 \\
\hline Hypotension & $1(5.0)$ & $2(100.0)$ & $2(11.1)$ & 0 & $2(22.2)$ & 0 & 0 \\
\hline Nasal congestion & 0 & 0 & 0 & 0 & $1(11.1)$ & $2(25.0)$ & 0 \\
\hline Nausea & 0 & 0 & $2(11.1)$ & 0 & $1(11.1)$ & 0 & 0 \\
\hline Rhinorrhea & $1(5.0)$ & 0 & 0 & $1(10.0)$ & 0 & 0 & 0 \\
\hline
\end{tabular}

IM = intramuscular; INP105 = olanzapine delivered by POD; ODT = oral disintegrating tablet; $O L Z$ = olanzapine; $P O D=$ precision olfactory delivery.

Figure 3: Percent of subjects achieving $\mathrm{T}_{\max }$ within designated time periods during 0-60 minutes of dose in the SNAP 101 study 32

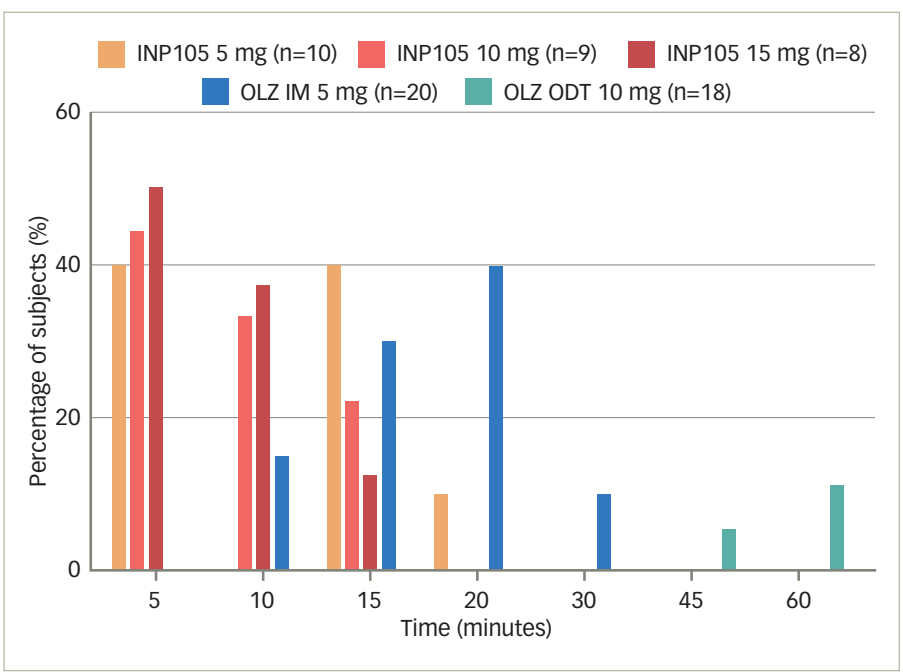

IM = intramuscular; INP105 = olanzapine delivered by POD; ODT = oral disintegrating tablet; $O L Z$ = olanzapine; $P O D=$ precision olfactory delivery.

SNAP 101 (INP105-101; ClinicalTrials.gov identifier: NCT03624322) was a randomized, placebo-controlled, two-period crossover study to evaluate the safety and tolerability of INP105. ${ }^{32}$ Three single ascending doses $(5,10$ and $15 \mathrm{mg})$ of INP105 were administered by the POD research device (Figure 1B) to compare the PK and pharmacodynamic effects of INP105 with OLZ IM (5 and $10 \mathrm{mg}$ ), OLZ oral disintegrating tablet (ODT) $10 \mathrm{mg}$, and placebo. It was not conducted in patients suffering from agitation, as the plan for development of INP105 is to "bridge" the PK data in healthy volunteers to that of IM OLZ, and if successful, obviating the need to conduct an efficacy trial. INP105 was well tolerated after a single dose (Table 1) in healthy volunteers
Figure 4: Mean Visual Analog Scale change from baseline seen over 6 hours in the SNAP 101 study in healthy volunteers ${ }^{32}$

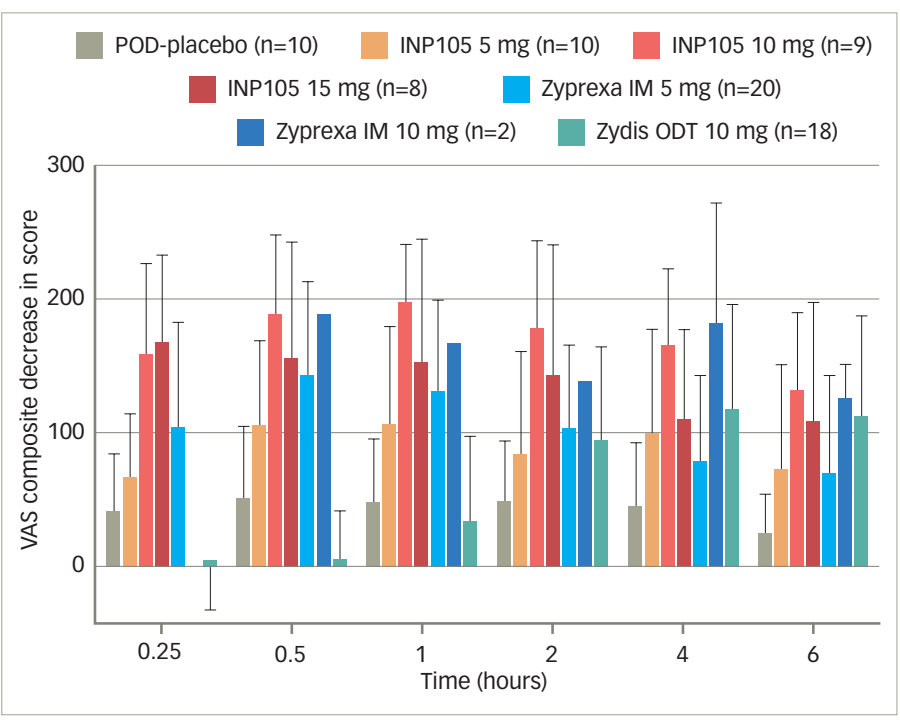

IM = intramuscular; INP105 = olanzapine delivered by POD; ODT = oral disintegrating tablet; $P O D=$ precision olfactory delivery; $V A S=$ visual analogue scale.

who had received either OLZ IM or OLZ ODT at least 14 days earlier in period 1. OLZ exposure with INP105 $5 \mathrm{mg}$ closely matched OLZ IM 5 mg, with most individual peak plasma levels occurring within 5-15 minutes with INP105, versus 10-20 minutes with OLZ IM 5 mg (Figure 3). ${ }^{32}$ Clinically meaningful calming effects were observed with INP105 versus placebo based on:

1) The Agitation and Calmness Evaluation Scale with changes of up to $\sim 2$ points over the first hour with INP105 that matched or exceeded the changes seen with OLZ IM $5 \mathrm{mg}$, whereas OLZ ODT $10 \mathrm{mg}$ and placebo did not substantially change from baseline in this first hour. The maximum changes over 6 hours were 0.6 (placebo), 2.1 (INP105 5 mg), 
Table 2: Treatment-emergent adverse events reported in the phase II INP103-201 single-dose trial conducted in patients with Parkinson's disease in an OFF episode ${ }^{33}$

\begin{tabular}{|c|c|c|c|c|c|}
\hline & \multicolumn{5}{|l|}{ Treatment } \\
\hline & \multicolumn{3}{|c|}{ Benserazide 25 mg with: } & \multirow{2}{*}{$\begin{array}{l}\text { INP107 } \\
70.0 \mathrm{mg} / 7.0 \mathrm{mg} \\
\text { Levodopa/carbidopa } \\
(\mathrm{n}=6)\end{array}$} & \multirow{2}{*}{$\begin{array}{l}\text { Placebo } \\
(n=8)\end{array}$} \\
\hline & $\begin{array}{l}\text { INP103 } 35 \mathrm{mg} \\
(\mathrm{n}=6)\end{array}$ & $\begin{array}{l}\text { INP103 } 70 \mathrm{mg} \\
(\mathrm{n}=6)\end{array}$ & $\begin{array}{l}\text { INP103 } 140 \mathrm{mg} \\
(\mathrm{n}=6)\end{array}$ & & \\
\hline Patients with $\geq 1 \mathrm{TEAE}$ & 5 (83.3\%) & $3(50.0 \%)$ & $4(66.7 \%)$ & $5(83.3 \%)$ & $5(62.5 \%)$ \\
\hline Upper respiratory tract irritation & - & $1(16.7 \%)$ & $1(16.7 \%)$ & - & - \\
\hline Rhinorrhea & - & - & $1(16.7 \%)$ & $1(16.7 \%)$ & - \\
\hline Sinus pain & - & - & $1(16.7 \%)$ & - & - \\
\hline Cough & - & - & - & - & $1(12.5 \%)$ \\
\hline Dry throat & - & - & - & $1(16.7 \%)$ & - \\
\hline Dyspnea & - & - & - & - & - \\
\hline Increased upper airway secretion & - & $1(16.7 \%)$ & - & - & - \\
\hline Nasal congestion & - & - & - & $1(16.7 \%)$ & - \\
\hline Nasal discomfort & - & - & - & - & $1(12.5 \%)$ \\
\hline Nasal dryness & - & - & $1(16.7 \%)$ & - & - \\
\hline Nasal edema & - & - & - & $1(16.7 \%)$ & - \\
\hline Paranasal sinus discomfort & - & - & - & $1(16.7 \%)$ & - \\
\hline Paranasal sinus hyposecretion & - & - & - & - & - \\
\hline Sneezing & $1(16.7 \%)$ & - & - & - & $1(12.5 \%)$ \\
\hline Headache & $2(33.3 \%)$ & - & - & - & - \\
\hline Somnolence & $1(16.7 \%)$ & $1(16.7 \%)$ & - & - & - \\
\hline Dizziness & $1(16.7 \%)$ & - & - & - & - \\
\hline Fatigue & - & - & - & - & $1(12.5 \%)$ \\
\hline Feeling abnormal & - & $1(16.7 \%)$ & - & - & - \\
\hline Hyperthermia & $1(16.7 \%)$ & - & - & - & - \\
\hline Hypertension & - & - & - & $1(16.7 \%)$ & $1(12.5 \%)$ \\
\hline Orthostatic hypotension & - & $1(16.7 \%)$ & - & - & - \\
\hline Rhinitis & - & - & - & $1(16.7 \%)$ & - \\
\hline Urinary tract infection & - & - & - & $1(16.7 \%)$ & - \\
\hline Joint noise & $1(16.7 \%)$ & - & - & - & - \\
\hline Muscle spasms & - & - & - & - & $1(12.5 \%)$ \\
\hline Tachycardia & - & - & - & - & $1(12.5 \%)$ \\
\hline Lacrimation increased & - & - & $1(16.7 \%)$ & - & - \\
\hline Procedural pain & - & - & $1(16.7 \%)$ & - & - \\
\hline
\end{tabular}

INP103 = levodopa delivered by POD; INP107 = carbidopa/levodopa delivered by POD; POD = precision olfactory delivery; TEAE = treatment-emergent adverse event.

2.9 (INP105 10 mg), 2.8 (INP105 15 mg), 1.9 (OLZ IM 5 mg), 2.8 (OLZ IM $10 \mathrm{mg}$ ) and 2.0 (OLZ ODT $10 \mathrm{mg}$ ).

2) The combined $300 \mathrm{~mm}$ Visual Analog Scale (exploring alert/drowsy; foggy/clear-headed and energetic/lethargic) showed rapid onset of effect with all three INP105 doses (of similar magnitude to OLZ 5 mg IM), substantially earlier than OLZ ODT and greater at all timepoints (out to 6 hours) compared with placebo (Figure 4).

3) The Digit Symbol Substitution Test (DSST) assessed response speed, sustained attention, visual spatial skills, and set shifting that corresponded to a series of digits (numerals) outlined on a test paper. Subjects were given 90 seconds to record the symbols associated with each numeral and the number of correctly substituted digits noted. There was a dose-dependent, desired, decrease in mean DSST scores representing a slowed response speed over the first 6 hours of testing for all doses of INP105 compared with placebo $(p<0.01)$. For INP105 $5 \mathrm{mg}$ and $10 \mathrm{mg}$ score changes were comparable to OLZ IM $5 \mathrm{mg}$ and greater than OLZ ODT $10 \mathrm{mg}$. Changes were maximal at 30 minutes (for 10 and $15 \mathrm{mg}$ ) and sustained for up to 6 hours. DSST score decreased at 2, 4, and 6 hours compared to placebo with OLZ ODT (but were not observed in the first hour with the ODT).

Thus, INP105 may represent a needle-free alternative for treating acutely agitated patients and could become a valuable option for selfor caregiver-administration in the psychiatric ward, nursing homes, the community (self-administered by self-aware patients), or the home environment. 


\section{Parkinson's disease-OFF episodes}

Patients with Parkinson's disease experience fluctuations of motor symptoms, even with optimized treatment. While levodopa is a standard of care for managing the dopamine-related symptoms of Parkinson's disease, most patients develop motor complications related to fluctuations in the levels of plasma levodopa over the course of their disease that may become debilitating and impact quality of life. An unmet need exists for rescue therapy that will rapidly and efficiently reverse OFF periods, especially in the early morning when the benefit of the previous evening's medication is at a nadir.

\section{POD levodopa and POD carbidopa/levodopa (in development)}

INP103 is a drug-device combination product containing levodopa delivered by the POD device. THOR 201 (INP103-201; ClinicalTrials. gov identifier: NCT03541356) was a randomized, double-blind, placebo-controlled, single-dose study that assessed the safety, tolerability, PK and pharmacodynamics of levodopa 35, 70, and $140 \mathrm{mg}$ administered by the POD device with a dopa decarboxylase inhibitor (DCI), for the treatment of early morning OFF episodes of Parkinson's disease. ${ }^{33}$ This was a proof of concept trial, albeit conducted in patients with Parkinson's disease, to ascertain if sufficient systemic levels of levodopa could be achieved to reverse OFF episodes. In the first three cohorts of eight subjects each (six active, two placebo), oral benserazide $25 \mathrm{mg} \mathrm{DCl}$ was dosed 60 minutes before INP103. As pharmaceutical development work progressed in parallel, a fourth cohort was added in early 2019, with INP107 in a 10:1 combination of levodopa/carbidopa at a dose of 70.0/7.0 mg. The levodopa absorption following early morning nasal administration of INP103 35, 70, and $140 \mathrm{mg}$ resulted in a median $\mathrm{T}_{\max }$ ranging between 45.5 and 60.5 minutes depending on dose, and 90.5 minutes for the combined carbidopa/levodopa (INP107) formulation. These corresponded to mean $\mathrm{C}_{\max }$ levels of levodopa of 294, 394 and 676 ng/mL for INP103 and 456 ng/mL for INP107.

Adverse events occurred at comparable rates among all dose cohorts, and all adverse events were mild (Table 2), except for one moderate treatment-emergent adverse event of urinary tract infection with no specific event reported by more than one subject in any cohort. Abnormal nasal examination findings were infrequently observed and were mild in severity. Most patients found the POD device comfortable to use and preferable compared with their current drug delivery method. The absorption of levodopa from the upper nasal space yielded adequate systemic exposure, which at $400 \mathrm{ng} / \mathrm{mL}$, has reversed daytime OFF motor symptoms, ${ }^{39}$ but the time to reach those concentrations was longer than desired and further work varying the ratio of carbidopa to levodopa is expected to greatly reduce that, optimize the PK profile, and provide clinical benefit, even for early morning OFF episodes, where absolute levodopa needs may be in the range $800-1,100 \mathrm{ng} / \mathrm{mL} .{ }^{40}$ The efficacy assessments, using the Movement Disorder Society Unified Parkinson's Disease Rating Scale (MDS-UPDRS), revealed mixed results with INP103, INP107 and placebo, and given the PK results (suggesting, at lower dose, the systemic levodopa level would not be expected to reverse morning OFF), small sample size, and 3:1 active:placebo randomization, may not represent a true pharmacological response.

\section{Summary}

While most drugs are administered as oral tablets, capsules or solutions, the need for rapid, effective systemic levels in disabling acute disorders, such as migraine, emergency situations (acute agitation), or in conditions affecting cognition and function (Parkinson's disease), make an easy to administer, non-injected, non-oral therapy much desired. In addition, the effectiveness of oral forms may be limited in patients suffering from dysphagia or GI motility problems, which are prevalent in migraine and Parkinson's disease. While a few drug-device products are approved for use in the USA to treat CNS disorders, the novel POD system is in clinical development for acute migraine, acute agitation, and OFF episodes in Parkinson's disease. The non-invasive POD system provides the most efficient nasal delivery of drugs, allowing it to outperform traditional nasal delivery products, as well as IM injection, with an optimized dose of active drug that results in few side effects. Delivery of drugs to the upper nasal space provides a useful, well-tolerated, and reliable alternative, and may be a welcome addition to the management of many CNS disorders. $\square$

\section{Article highlights}

- Solid oral dosage forms account for up to $75 \%$ of prescriptions from healthcare providers. However, their effectiveness may be limited by features of the disease/condition.

- While injectable formulations may be an option for some central nervous system (CNS) disorders, injections are poorly accepted by most patients, and usually require healthcare practitioner administration.

- A need exists for non-oral dosage formulations as alternatives to treat CNS conditions, such as acute migraine, acute agitation associated with bipolar disorder or schizophrenia, and OFF episodes in Parkinson's disease.

- Nasal administration has been underutilized for drug delivery and has often had inconsistent clinical results; however, new delivery systems that address the shortcomings of traditional delivery systems may unlock the benefits of nasal delivery by avoiding first-pass hepatic metabolism, providing a rapid onset of activity, achieving therapeutic plasma levels, and improving patient convenience and ease-of-use.

- POD delivery technology delivers drugs to the upper nasal space and several POD enabled products are in late-stage development for treating CNS disorders.
1. Schiele JT, Quinzler R, Klimm HD, et al. Difficulties swallowing solid oral dosage forms in a general practice population: prevalence, causes, and relationship to dosage forms. Eur I Clin Pharmacol. 2013;69:937-48.

2. Stegemann $S$, Gosch M, Breitkreutz J. Swallowing dysfunction and dysphagia is an unrecognized challenge for oral drug therapy. Int J Pharm. 2012;430:197-206.

3. Ferrari A, Tiraferri I, Neri L, Sternieri E. Why pharmacokinetic differences among oral triptans have little clinical importance: a comment. J Headache Pain. 2011;12:5-12.

4. Logrippo S, Ricci G, Sestili M, et al. Oral drug therapy in elderly with dysphagia: between a rock and a hard place! Clin Interv Aging. 2017:12:241-51.

5. Tannenbaum C, Sheehan NL. Understanding and preventing drug-drug and drug-gene interactions. Expert Rev Clin Pharmacol 2014;7:533-44

6. Lau ETL, Steadman KJ, Cichero JAY, Nissen LM. Dosage form modification and oral drug delivery in older people. Adv Drug Deliv Rev. 2018:135:75-84

7. Láinez MJ, García-Casado A, Gascón F. Optimal management of severe nausea and vomiting in migraine: improving patient outcomes. Patient Relat Outcome Meas. 2013;4:61-73.

8. Aurora SK, Kori SH, Barrodale P, et al. Gastric stasis in migraine more than just a paroxysmal abnormality during a migraine attack. Headache. 2006;46:57-63.

9. Aurora SK, Papapetropoulos S, Kori SH, et al. Gastric stasis in migraineurs: etiology, characteristics, and clinical and therapeutic implications. Cephalalgia. 2013;33:408-15.
10. Pini LA, Brovia D. Different characteristics of triptans. $J$ Headache Pain. 2004;5:S109-11.

11. Diamond S, Freitag FG, Feoktistov A, Nissan G. Sumatriptan 6 mg subcutaneous as an effective migraine treatment in patients with cutaneous allodynia who historically fail to respond to oral triptans. $J$ Headache Pain. 2007:8:13-8.

12. Suttrup I, Warnecke T. Dysphagia in Parkinson's disease. Dysphagia. 2016;31:24-32.

13. Reisch T, Beeri S, Klein G, et al. Comparing attitudes to containment measures of patients, health care professionals and next of kin. Front Psych. 2018:9:1-8

14. Zeller SL, Citrome L. Managing agitation associated with schizophrenia and bipolar disorder in the emergency setting. West J Emerg Med. 2016;17:165-72. 
15. Musumeci T, Bonaccorso A, Puglisi G. Epilepsy disease and nose-to-brain delivery of polymeric nanoparticles: an overview. Pharmaceutics. 2019;11:118.

16. Lochhead J, Thorne R. Intranasal delivery of biologics to the central nervous system. Adv Drug Deliv Rev. 2012;64:614-28.

17. Erdo $F, B$ Bors $L A$, Farkas $D$, et al. Evaluation of intranasal delivery route of drug administration for brain targeting. Brain Res Bull. 2018:143:155-70

18. Shipley MT. Transport of molecules from nose to brain: transneuronal anterograde and retrograde labeling in the rat olfactory system by wheat germ agglutinin-horseradish peroxidase applied to the nasal epithelium. Brain Res Bull. 1985;15:129-42.

19. Sabin AB, Olitsky PK. Influence of path of infection on pathology of olfactory bulbs in experimental poliomyelitis. Proc SOC Exp Biol Med. 1936;35:300-1.

20. Sabin AB, Olitsky PK. Influence of host factors on neuroinvasiveness of vesicular stomatitis virus: I. Effect of age on the invasion of the brain by viruses instilled in the nose. J Exp Med. 1937;66:15-34.

21. Arvidson B. A review of axonal transport of metals. Toxicology. 1994;88:1-14.

22. Danielyan L, Schafer R, von Ameln-Mayerhofer A, et al. Intranasal delivery of cells to the brain. Eur I Cell Biol. 2009;88:315-24.

23. van Velthoven CT, Kavelaars A, van Bel F, Heijnen CJ. Nasal administration of stem cells: a promising novel route to treat neonatal ischemic brain damage. Pediatr Res. 2010;68:419-22

24. Rapoport AM, Bigal ME, Tepper SJ, Sheftell FD. Intranasal medications for the treatment of migraine and cluster headache CNS Drugs. 2004;18:671-85.

25. Lipton RB, Buse DC, Serrano D, et al. Examination of unmet treatment needs among persons with episodic migraine: results of the American Migraine Prevalence and Prevention (AMPP) study. Headache. 2013;53:1300-11.

26. Hazard E, Munakata J, Bigal ME, et al. The burden of migraine in the United States: current and emerging perspectives on disease management and economic analysis. Value Health 2009;12:55-64.

27. Shrewsbury SB, Jeleva M, Satterly KH, et al. STOP 101: A phase 1 , randomized, open-label, comparative bioavailability study of INP104, dihydroergotamine mesylate (DHE) administered intranasally by a 1123 Precision Olfactory Delivery (POD ${ }^{\oplus}$ ) device, in healthy adult subjects. Headache. 2019:59:394-409.

28. Hoekman J, Brunelle A, Hite M, et al. SPECT imaging of direct nose-to-brain transfer of MAG-3 in man. Poster W4009 presented at the American Association of Pharmaceutical Scientists Annual Meeting, San Antonio, TX, USA, November 10-14, 2013.

29. Olafsson DR, Gizurarson S. Access to the olfactory region. Proc Control Release Bioact Mater. 2000;27:6318.

30. Hoekman J, Brunelle A, Hite M, et al. Usability and tolerability study in human subjects with a novel precision olfactory delivery (POD) device. Poster M1050 presented at the American Association of Pharmaceutical Scientists Annual Meeting. Association of Pharmaceutical Scientists Annu

31. Shrewsbury SB, Jeleva M, Hocevar-Trnka J, Swardstrom M. STOP 301: Open-label safety and tolerability of chronic intermittent usage for 24/52 weeks of INP104 [nasal dihydroergotamine mesylate (DHE) administered by Precision Olfactory Delivery (POD ${ }^{\circledR}$ ) Device] in migraine headache. Presented at the International Headache Congress, Dublin Ireland, September 5-8, 2019. Cephalalgia. 39(Suppl. 1):208-9.

32. Shrewsbury SB, Hocevar-Trnka J, Satterly KH, et al. The SNAP 101 double-blind, placebo/active-controlled, safety, pharmacokinetic, and pharmacodynamic study of INP105 (nasal olanzapine) in healthy adults. J Clin Psychiatry. 2020;81:19m13086.

33. Shrewsbury SB, Campbell J, Swardstrom M, et al. THOR 201: A proof-of-concept study assessing safety, tolerability, pharmacokinetics and pharmacodynamics of $\mathrm{L}$-dopa delivered pharmacokinetics and pharmacodynamics of $L$-dopa delivered
by Impel's Precision Olfactory Delivery (PODTM) to Parkinson's disease patients in a morning OFF episode (in the presence of dopa decarboxylase inhibitor) [abstract]. Mov Disord 2019;34(Suppl. 2). Available at: www.mdsabstracts.org/abstract/ thor-201-a-proof-of-concept-study-assessing-safety-tolerability pharmacokinetics-and-pharmacodynamics-of-I-dopa-deliveredby-impels-precision-olfactory-delivery-podtm-to-parkinson/ (accessed July 7, 2020)

34. Shrewsbury SB, Hoekman J, Jeleva M. Patient acceptability of a novel upper nasal delivery system for dihydroergotamine mesylate using the Precision Olfactory Delivery (POD $\left.{ }^{\circledR}\right)$ device mesylate using the Precision Olfactory Delivery (POD ${ }^{\circledR}$ ) dev
- results from the open-label STOP 301 trial. Presented at - results from the open-label STOP 301 trial. Presented at
the 2020 American Headache Society (AHS) Virtual Annual Scientific Meeting. Available at: https://impelnp.com/wpcontent/uploads/2020/06/AHS-2020-STOP-301.PAQ-Poster FINAL_05-26-20-1.pdf (accessed July 7, 2020).

35. Dundar Y, Greenhalgh J, Richardson M, et al. Pharmacological treatment of acute agitation associated with psychotic and bipolar disorder a systematic review and meta-analysis. Hum Psychopharmacol Clin Exp. 2016;31:268-85.

36. Garriga M, Pacchiarotti I, Kasper S, et al. Assessment and management of agitation in psychiatry: expert consensus. World I Biol Psychiatry. 2016;17:86-128.

37. Baio J, Wiggins L, Christensen DL, et al. Prevalence of autism spectrum disorder among children aged 8 years - autism and developmental disabilities monitoring network, 11 sites, United States, 2014. MMWR Surveill Summ. 2018:67:1-23.

38. Chaidez V, Hanson RL, Hertz-Picciotto I. Gastrointestinal problems in children with autism, developmental delays or typical development. J Autism Dev Disord. 2014;44:117-27.

39. Lipp MM, Batycky R, Moore J, et al. Preclinical and clinical assessment of inhaled levodopa for OFF episodes in Parkinson's disease. Sci Trans/ Med. 2016;8:1-10.

40. LeWitt PA. Levodopa therapy for Parkinson's disease: pharmacokinetics and pharmacodynamics. Mov Disord. 2015:30:64-72 